\title{
Equine grass sickness (a multiple systems neuropathy) is associated with alterations in the gastrointestinal mycobiome
}

Bruce C. McGorum' ${ }^{*}$, Zihao Chen², Laura Glendinning ${ }^{1}$, Hyun S. Gweon ${ }^{3}$, Luanne Hunt ${ }^{1}$, Alasdair Ivens², John A. Keen ${ }^{1}$, R. Scott Pirie ${ }^{1}$, Joanne Taylor ${ }^{4}$, Toby Wilkinson ${ }^{1}$ and Gerry McLachlan ${ }^{1}$

\begin{abstract}
Background: Equine grass sickness (EGS) is a multiple systems neuropathy of grazing horses of unknown aetiology. An apparently identical disease occurs in cats, dogs, rabbits, hares, sheep, alpacas and llamas. Many of the risk factors for EGS are consistent with it being a pasture mycotoxicosis. To identify potential causal fungi, the gastrointestinal mycobiota of EGS horses were evaluated using targeted amplicon sequencing, and compared with those of two control groups. Samples were collected post mortem from up to 5 sites in the gastrointestinal tracts of EGS horses (EGS group; 150 samples from 54 horses) and from control horses that were not grazing EGS pastures and that had been euthanased for reasons other than neurologic and gastrointestinal diseases (CTRL group; 67 samples from 31 horses). Faecal samples were also collected from healthy control horses that were co-grazing pastures with EGS horses at disease onset (CoG group; 48 samples from 48 horses).

Results: Mycobiota at all 5 gastrointestinal sites comprised large numbers of fungi exhibiting diverse taxonomy, growth morphology, trophic mode and ecological guild. FUNGuild analysis parsed most phylotypes as ingested environmental microfungi, agaricoids and yeasts, with only $1 \%$ as gastrointestinal adapted animal endosymbionts. Mycobiota richness varied throughout the gastrointestinal tract and was greater in EGS horses. There were significant inter-group and inter-site differences in mycobiota structure. A large number of phylotypes were differentially abundant among groups. Key phylotypes $(n=56)$ associated with EGS were identified that had high abundance and high prevalence in EGS samples, significantly increased abundance in EGS samples, and were important determinants of the inter-group differences in mycobiota structure. Many key phylotypes were extremophiles and/or were predicted to produce cytotoxic and/or neurotoxic extrolites.
\end{abstract}

Conclusions: This is the first reported molecular characterisation of the gastrointestinal mycobiota of grazing horses. Key phylotypes associated with EGS were identified. Further work is required to determine whether neurotoxic extrolites from key phylotypes contribute to EGS aetiology or whether the association of key phylotypes and EGS is a consequence of disease or is non-causal.

Keywords: Equine grass sickness, Equine dysautonomia, Gastrointestinal mycobiota, Fungi, Multiple systems neuropathy

\footnotetext{
*Correspondence: Bruce.mcgorum@ed.ac.uk

${ }^{1}$ Royal (Dick) School of Veterinary Studies and The Roslin Institute, Easter Bush Veterinary Centre, University of Edinburgh, Roslin, Midlothian EH25 9RG, UK

Full list of author information is available at the end of the article
}

\section{Background}

Equine grass sickness (EGS) is a predominantly fatal, multiple systems neuropathy of grazing horses that kills approximately $1-2 \%$ of horses grazing affected premises in the United Kingdom annually [1-3]. It is characterised 
by chromatolysis, degeneration and loss of enteric neurons, peripheral and central autonomic neurons, neurons in specific brain stem nuclei and spinal cord somatic efferent lower motor neurons $[3,4]$. The predominant clinical features of EGS are attributable to paralysis of the entire gastrointestinal (GI) tract caused by severe enteric neuropathy $[1,2]$. Striking similarities in the clinico-pathological features of EGS with multiple systems neuropathies of cats (feline dysautonomia), dogs (canine dysautonomia), hares (leporine dysautonomia), rabbits, alpacas, llamas and sheep (abomasal emptying defect) suggest these represent a specific disease entity with a common, but currently unknown, aetiology [511]. While some evidence supports an association with Clostridium botulinum type C/D [1, 2, 12-14], many risk factors for EGS are consistent with it being caused by a neurotoxic extrolite from a pasture-derived fungus $[1,15]$. Equids, being monogastric animals, are considered to be more sensitive to dietary fungi and mycotoxins than ruminants [16]. EGS has a strong association with grazing, particularly on certain pastures [17]. Pasturerisk factors for EGS include sand/loam rather than chalk soils, high soil nitrogen, low soil $\mathrm{Cu}$ and $\mathrm{Zn}$ content, and pasture disturbance [18-20]. As for other pasture mycotoxicoses, EGS is strongly seasonal, with peak incidence occurring during spring and early summer [17-21] when many fungi are actively growing [22]. The seasonality may reflect climate-level risk factors for EGS, including cooler, drier weather and irregular ground frosts [17-19], which may favour fungal growth and extrolite elaboration [19], and/or the link between the growth of causal fungi on plants and the seasonal pattern of plant growth [23]. The first description of EGS in 1906 has been linked to the dramatic pasture improvements made in Scotland at that time, including drainage, ploughing, a change from permanent to rotational grazing and introduction of new grass seed mixtures which may have been contaminated with fungal spores [24]. Previous studies identified Acremonium and Fusarium on plants from all studied EGS fields in Scotland and Patagonia [24]. Comparison of the intestinal mycobiota of EGS and control horses using culture-based techniques identified a wide variety of fungi including mycotoxin producing species in EGS and control horses, but EGS was not associated with a particular mycotoxigenic fungus [22]. These authors acknowledged significant study limitations, in particular the inability to isolate and identify the wide variety of fungi present in the equine GI tract. Mycotoxicosis may also account for the depletion of the plasma sulphur amino acids cyst[e] ine and methionine in EGS and feline dysautonomia [25, 26], since these strong nucleophiles reduce, detoxify and facilitate the excretion of electrophilic compounds and free radicals derived from dietary toxins including fungal extrolites [27, 28]. EGS was not induced in experimental horses by intra-gastric administration of cultures of fungi obtained from EGS plants including a Basidiomycete, Rhizopus, Mucoraceae and a putative Phoma [29], nor by feeding Acremonium spp.-colonised Festuca grass [30] or ergots [31].

The aim of this study was to investigate whether EGS is associated with ingestion of mycotoxin-producing fungi. Targeted amplicon sequencing of the internal transcribed spacer 1 (ITS1) of the fungal ribosomal RNA gene cluster was used to compare the mycobiota within the stomach, ileum, caecum, colon and faeces of EGS and control grazing horses, and within the faeces of healthy co-grazing control horses. Co-grazing control horses remain clinically healthy but have increased serum concentrations of acute phase proteins [32] consistent with sub-clinical exposure to the toxin which causes EGS. Metabarcoding analysis of the mycobiota throughout the gastro-intestinal (GI) tract of grazing horses has not been previously reported. We hypothesised that detection of putative causal fungi was more likely achieved by examination of the GI mycobiota of grazing EGS horses than by examination of mycobiota of soil and herbage samples collected from the horses' fields. This study identified key phylotypes $(n=56)$ that had increased abundance and high prevalence in EGS samples, and which were important determinants of the inter-group differences in mycobiota structure. Further work is required to determine whether neurotoxic extrolites from these key phylotypes have a causal role in EGS, or whether their association with EGS is coincidental.

\section{Results}

\section{Sample groups}

There was no significant inter-group difference in sex (Chi-square test $P=0.44$ ) or month of sample collection (paired t-test $P=0.08$ ). EGS horses (median 5 years, inter-quartile range 4.0-7.5) were significantly younger than CoG (8 years, 5.0-10.3; Mann-Whitney $P=0.011$ ) and control (16 years, $12.0-25.0 ; P<0.0001)$ horses.

\section{Sequence data}

A total of $23,590,409$ high quality sequences, representing 13,204 OTUs and 2816 phylotypes of diverse taxonomy, were acquired from 265 GI samples (Additional file 1; Table S1). Samples had a minimum of 5122 and maximum of 2,152,731 sequences (median 60,768; interquartile range 32,600-106,289; mean 89,020). Rarefaction curves of phylotype richness for individual samples (Additional file 1; Fig. S1) indicated adequate sample size to capture the complex and diverse mycobiota structure. 


\section{Negative and positive controls}

Negative (nuclease-free water) and positive mock fungal community controls were run in parallel with samples. Mock community control 1 was a mixed microbial population which included rDNA from Saccharomyces cerevisiae and Cryptococcus neoformans. Mock community 2 comprised rDNA extracted from 10 fungi (Alternaria infectoria, Coniochaeta lignicola, Didymella rumicicola, Mycosphaerella tassiana, Penicillium pagulum, Pyrenochaetopsis pratorum, Vishniacozyma victoriae, Xylaria longipes, G_Eutypella and G_Fusarium) which colonised grasses collected from equine pastures within the geographical area from which the equine GI samples were collected. Negative controls $(n=16)$ had low amplicon counts (mean 1486, range 32-9672), except 3 samples that were contaminated with Alternaria infectoria and Mycosphaerella tassiana. All 12 taxa in the two mock communities were identified in all mock community samples. $2 / 10$ mock community 2 and $4 / 7$ mock community 1 samples had total amplicon counts $<5000$ and were removed from the analysis. Saccharomyces cerevisiae and Cryptococcus neoformans were identified in all 3 mock community 1 samples, but the former was in low abundance. All 10 fungi were identified in all mock community 2 samples. Some taxa in mock community 2, including Coniochaeta lignicola, were represented by a single OTU, while others, including Vishniacozyma victoriae, had multiple OTUs. Of the 13,204 OTUs, 53 were likely contaminants; as this analysis was done retrospectively the contaminants were not removed from the subsequent analysis. None of the contaminant OTUs contributed to the key phylotypes.

\section{FUNGuild analysis}

FUNGuild analysis parsed 2460 of the 2816 (87\%) phylotypes into 20 growth morphologies, 26 ecological guilds and 3 tropic modes. Some fungi were parsed into multiple categories. Growth morphologies were predominantly microfungi (32.7\%), null (30.2\%), agaricoid (14.7\%) and yeast (9.5\%) (Additional file 1; Table S2). Tropic mode was predominantly saprotrophs $(75.3 \%)$, with smaller proportions of pathotrophs (35.0\%) and symbiotrophs (25.9\%) (Additional file 1; Table S3). In terms of ecological guild, most phylotypes were assigned to undefined saprotrophs (55.1\%), plant pathogens (23.2\%), wood saprotrophs (16.7\%), endophytes (12.8\%), animal pathogens (11.8\%), fungal parasites (10.3\%), ectomycorrhizal (8.9\%), soil saprophytes $(7.4 \%)$ and dung saprotrophs (6.8\%) (Additional file 1; Table S4). Only 1\% of phylotypes were animal endosymbionts including Neocallimastigaceae.

\section{Dominant fungal taxa}

Taxa were identified to kingdom $(98.0 \%, n=3)$, phylum $(71.8 \%, n=18)$, class $(66.6 \%, n=46)$, order $(64.5 \%$, $n=143)$, family $(55.4 \%, n=355)$, genus $(50.7 \%, n=1004)$ and species $(40.1 \%, n=2317)$ levels. Visual taxonomic summaries for all samples are presented for phylum, class (Fig. 1), order and family (Additional file 1: Fig. S2). While most taxa were $k_{-}$Fungi, there were also protists ( $\mathrm{k} \_$Rhizaria) and taxa unidentified at kingdom level. The dominant phyla were Ascomycota, Basidiomycota, Mortierellomycota and Neocallimastigomycota (Fig. 1A). Dominant classes were Dothideomycetes, Eurotiomycetes, Leotiomycetes, Neocallimastigomycetes, Saccharomycetes, Sordariomycetes, Tremellomycetes and Wallemiomycetes (Fig. 1B). Dominant orders were Capnodiales, Eurotiales,
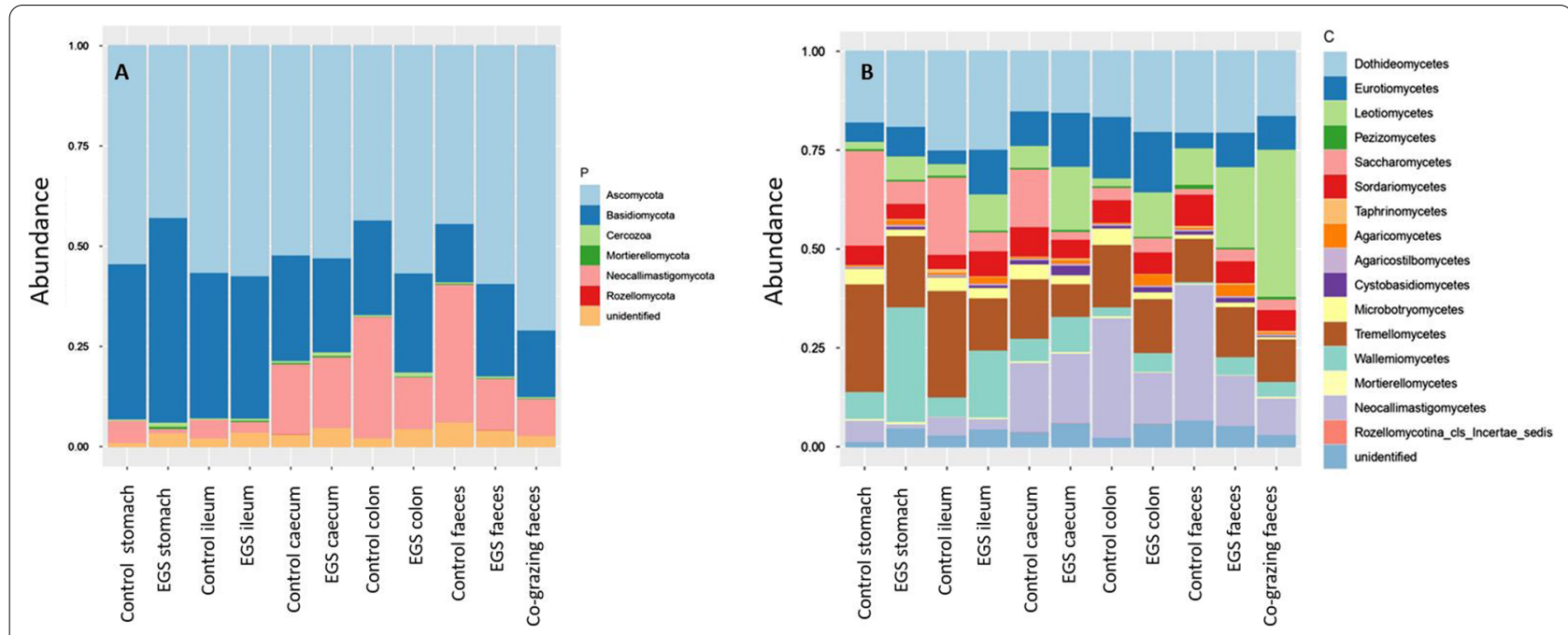

Fig. 1 Taxonomy plots showing relative abundance of taxa at A phylum and $\mathbf{B}$ class levels. Data are filtered at $0.05 \%$ abundance threshold 
Filobasidiales, Neocallimastigales, Pleosporales, Saccharomycetales and Thelebolales (Additional file 1: Fig. S2a). Dominant families were Aspergillaceae, Bulleribasidiaceae, Filobasidiaceae, Neocallimastigaceae, Phaffomycetaceae, Sporormiaceae, Thelebolaceae and Wallemiaceae (Additional file 1: Fig. S2b). Dominant genera were Aspergillus, Naganishia, Piromyces, Preussia, Thelebolus, Vishniacozyma, Wallemia and Wickerhamomyces. Dominant species were Aspergillus proliferans, Vishniacozyma victoriae, Wallemia muriae, W. sebi and Wickerhamomyces anomalus.

\section{Fungal species richness and diversity (alpha-diversity)}

Mycobiota richness (Chao 1) differed significantly across all 5 GI sites (Kruskal Wallis; $P=0.0084$ ) and among groups (Fig. 2; Additional file 1; Table S5). Mycobiota richness was higher in faeces versus proximal (stomach and ileum) GI sites. Pairwise inter-site comparisons indicated richness was higher in faeces than in stomach (Chao1 $P=0.00364$ ) and ileum (Chao1 $P=0.00190)$ (Additional file 1; Table S5). Diversity (Inverse Simpson) was not significantly different across GI sites (Kruskal Wallis; $P=0.1914$ ).
Inter-group comparisons demonstrated increases in Chao1 in caecum and colon samples from EGS horses versus those from CTRL horses (Table 1), and increases in Inverse Simpson index in EGS colon samples versus those from controls and in EGS faeces samples versus those from CoG horses (Table 1).

\section{Inter-site and inter-group differences in mycobiota structure (Beta-diversity)}

PLS-DA and weighted UniFrac distance analysis (Betadiversity) identified significant differences in mycobiota structure among GI sites (Fig. 3; Additional file 1; Table S6) and among groups (Fig. 4; Additional file 1; Table S7), at phylotype level. There were significant intersite dissimilarities in mycobiota structure in EGS horses, between stomach versus colon $(P=0.027)$ and faeces $(P=0.0001)$, and between ileum versus faeces $(P=0.01)$ (Additional file 1; Table S6). Inter-group comparisons of EGS versus CTRL at each GI site identified dissimilarity overall $(P=0.023)$ and in stomach samples $(P=0.001)$ (Figs. 4 and 5; Additional file 1: Table S7). There was also significant dissimilarity in structure of mycobiota in faeces samples from EGS versus CoG $(P=0.008)$ and CoG versus CTRL $(P=0.006)$.

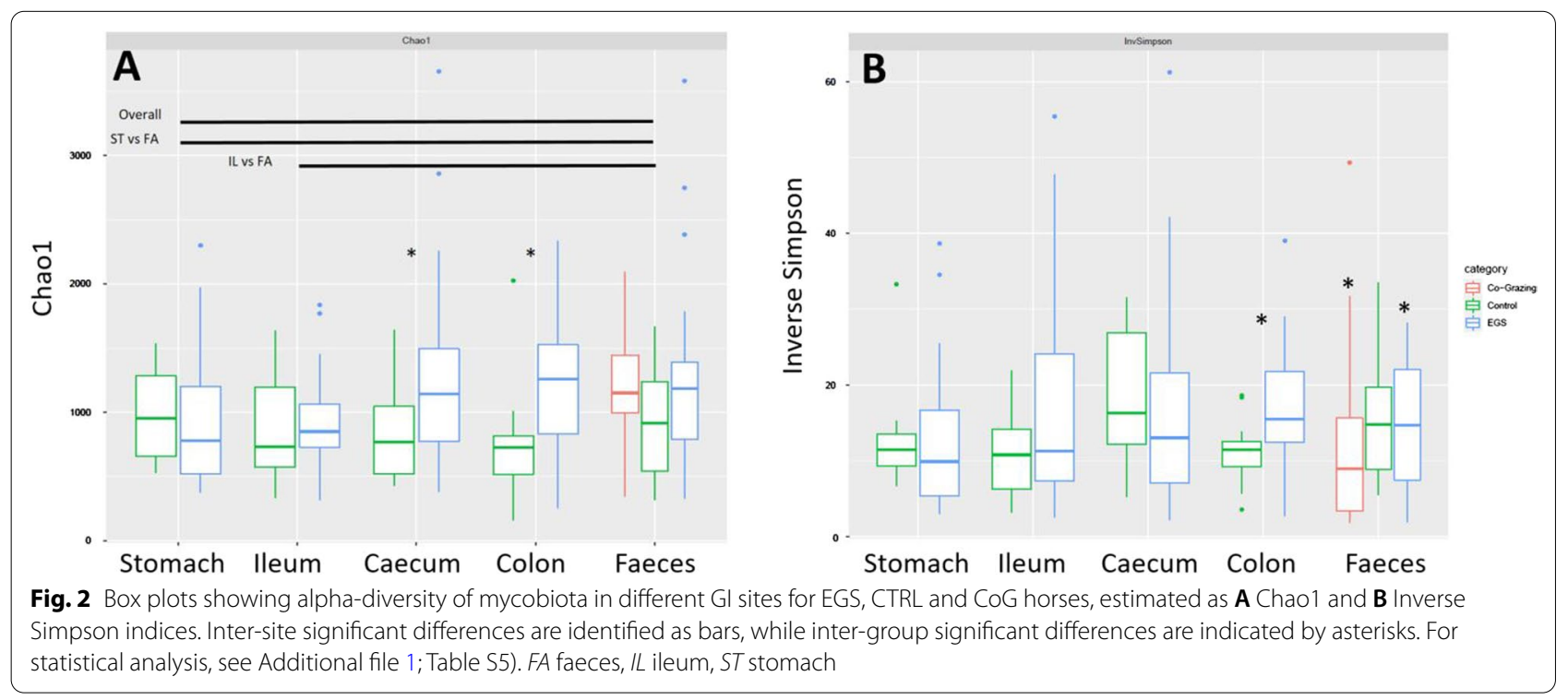

Table 1 Inter-group comparisons of alpha-diversity indices at each GI site

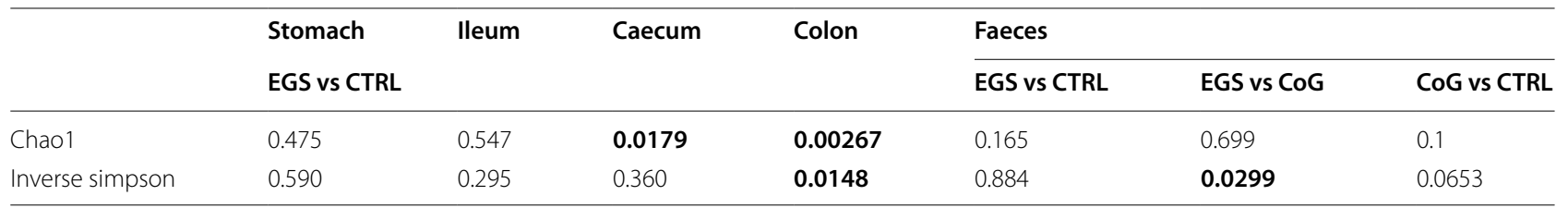

$P$ values indicating significantly higher alpha-diversity in EGS samples are in bold 

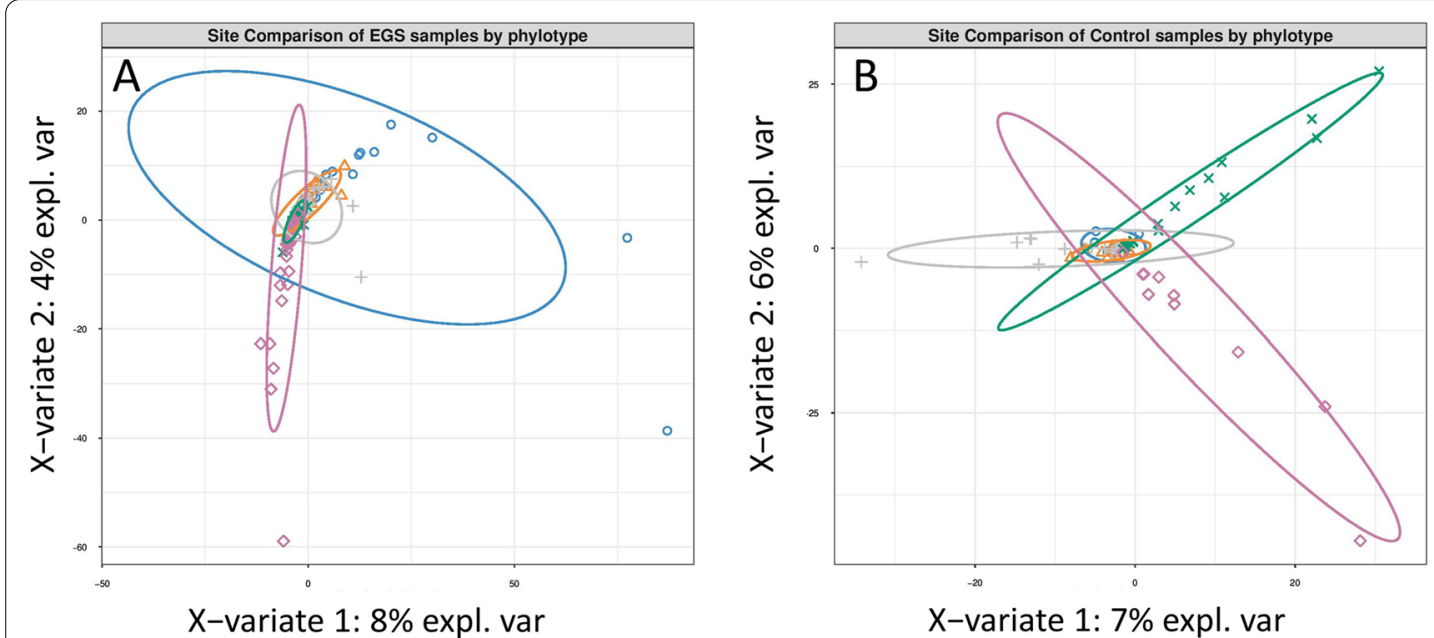

Legend

- caecum

$\triangle$ colon

+ faeces

$X$ ileum

stomach

Fig. 3 PLS-DA plots showing clustering of samples by GI site in A EGS and B CTRL groups, at phylotype level. Confidence ellipses show 95\% confidence level. For statistical analysis, see Additional file 1; Table S6

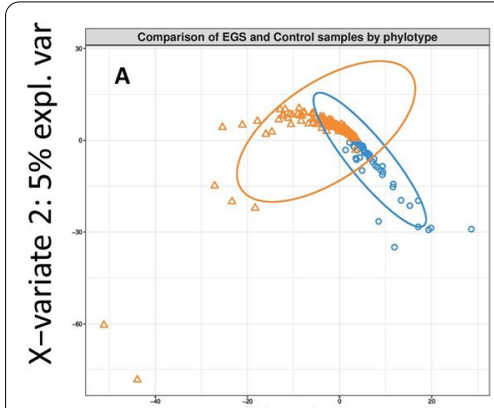

X-variate 1: 5\% expl. var

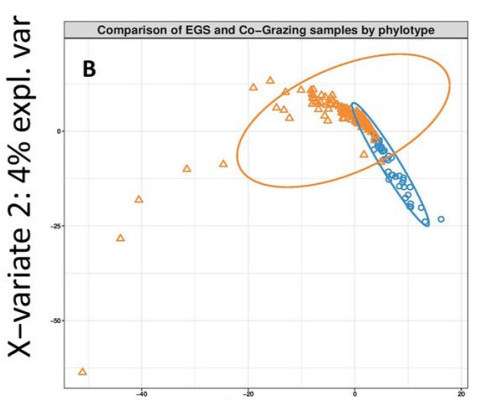

X-variate 1: 7\% expl. var

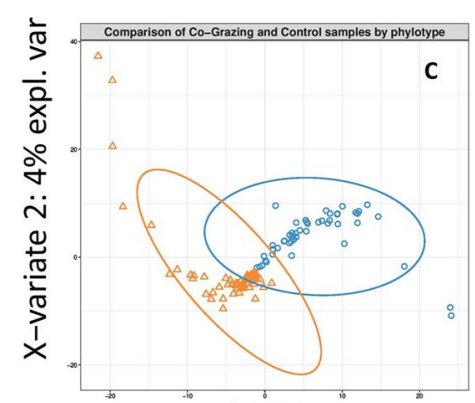

X-variate 1: 4\% expl. var

Fig. 4 Partial Least Squares Discriminant Analysis (PLS-DA) plots showing separate clustering of all samples from A EGS vs CTRL, B EGS vs CoG and C CoG vs CTRL, at phylotype level. Weighted UniFrac distance analysis identified significant inter-group dissimilarities (Additional file 1; Table S7). Confidence ellipses show 95\% confidence level

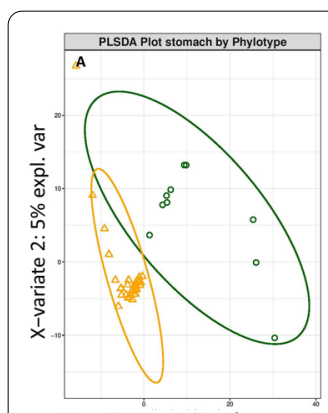

X-variate 1: $6 \%$ expl. var

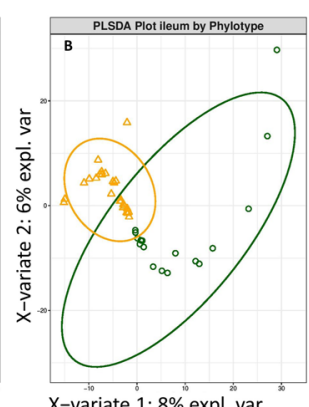

X-variate 1: $8 \%$ expl. var

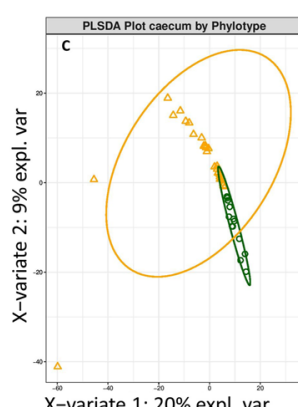

X-variate 1: $20 \%$ expl. var
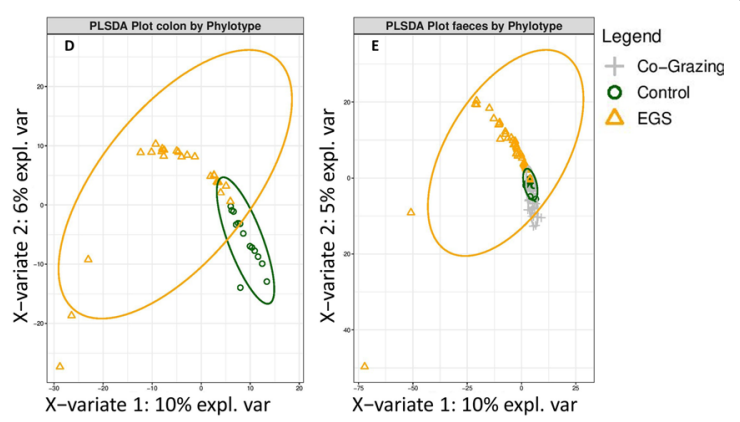

Fig. 5 Partial Least Squares Discriminant Analysis (PLS-DA) plots of samples from EGS, CTRL and CoG groups, analysed separately for $\mathbf{A}$ stomach, B ileum, C caecum, D colon and $\mathbf{E}$ faeces. Weighted UniFrac distance analysis identified significant inter-group dissimilarities (Additional file 1; Table S7). Confidence ellipses show 95\% confidence level 
Identification of differentially abundant phylotypes using inter-group DESeq2 analysis

DESeq2 inter-group analysis identified many differentially abundant phylotypes at Phylum, Class, Order, Family, Genus and phylotype levels (Additional file 1; Table S8 \& Additional file 2). Significantly more of the differentially abundant taxa were more abundant in EGS vs CTRL and in CoG vs CTRL groups, at each taxonomic level (paired t test, $P=0.001)$. Phylotypes with increased $(n=877)$ and decreased $(n=488)$ abundance, in at least one GI site, in the EGS group (versus CoG and CTRL groups) and in CoG versus CTRL groups are listed in Additional file 2.

Genera with $\geq 4$ phylotypes that had decreased abundance in EGS were Acremonium, Alternaria, Aspergillus, Candida, Cladosporium, Fusarium, Neoascochyta, Penicillium, Ramularia, Talaromyces, Taphrina (all Ascomycota), Coprinellus, Coprinopsis, Cutaneotrichosporon, Cystofilobasidium, Dioszegia, Filobasidium, Leucosporidium, Naganishia, Papiliotrema, Psathyrella, Rhodotorula, Udeniomyces, Vishniacozyma (all Basidiomycota) and Mortierella (Mortierellomycota). Of the phylotypes with decreased abundance in EGS horses, the ten with highest variable importance in projection (VIP) scores were Mycosphaerella tassiana (VIP 3.20), Wickerhamomyces anomalus (2.99), Filobasidium oeirense (2.87), Trichosporon insectorum (2.83), Cladosporium grevilleae (2.77), Trichosporon lactis (2.57), Sporobolomyces roseus (2.56), G_Fusarium (2.47), O_Hypocreales (2.41) and Cladosporium ramotenellum (2.40) (Additional file 2).

\section{Key phylotypes associated with EGS}

Key phylotypes ( $n=56$; Table 2 ; Additional file 3 ) associated with EGS, and which could have a potential role in EGS aetiology, were identified. These had; a) high abundance in EGS samples ie being within the top 50\% quantile of abundant phylotypes, b) high prevalence in EGS samples ie present in $>50 \%$ of EGS samples included in each paired comparison, c) a VIP score $>1.5$ and significantly increased abundance $[\mathrm{Padj}<0.05, \log 2$ fold change $>0$ ] in EGS samples in any comparison of EGS versus CTRL (overall, stomach, ileum, caecum, colon, faeces) or EGS versus CoG (faeces) samples. Key phylotypes comprised fungi of diverse taxonomy. All belonged to two phyla, namely Ascomycota $(n=28)$ and Basidiomycota $(n=25)$, except one from Mortierellomycota, one from Chytridiomycota and one unidentified at phylum level. Key phylotypes belonged to the ten dominant classes, except for Neocallimastigomycetes. Five key phylotypes were unidentified or incertae sedis at order level, while the remainder belonged to 24 orders. Fourteen key phylotypes were unidentified or incertae sedis at family level, while the remainder belonged to 27 families. Eighteen key phylotypes were unidentified or incertae sedis at genus level, while the remainder belonged to 29 genera. Thirty three key phylotypes were identified at species level.

FUNGuild classifications were available for 35 of 56 (63\%) key phylotypes. Growth morphologies were predominantly microfungi (37.1\%), null (31.4\%), yeasts (17.1\%) and agaricoids (11.4\%)(Additional file 1; Table S2; Additional file 4). Tropic mode was predominantly saprotroph (91.4\%), with smaller proportions of pathotrophs (34.3\%) and symbiotrophs (28.6\%)(Additional file 1; Table S3; Additional file 4). In terms of ecological guild, most phylotypes were assigned to undefined saprotrophs (71.4\%), fungal parasites (22.8\%), dung saprotrophs (20.0\%), endophytes (17.1\%), plant pathogens $(14.3 \%)$, animal pathogens (14.3\%) soil saprotrophs (11.4\%), wood saprotrophs (11.4\%), and plant saprotrophs (8.6\%)(Additional file 1; Table S4; Additional file 4).

The structure of the faecal mycobiota of CoG horses differed significantly from those of EGS and CTRL horses (Additional file 2). Of the 56 key phylotypes associated with EGS, 29 were more abundant in faeces of EGS vs CoG groups, while 9 were significantly more abundant in faeces of CoG vs CTRL horses (Additional File 2).

\section{Discussion}

Grazing horses have a very rich and diverse GI mycobiota This is the first reported molecular characterisation of the mycobiota throughout the GI tract of grazing horses. All horses, at all 5 GI sites, had a very rich and diverse mycobiota, evidenced by the overall detection of 13,204 OTUs and 2816 phylotypes. The majority of taxa were identified to genus level, but only $40.1 \%$ were identified to species level. Dominant phyla, in terms of abundance, were Ascomycota, Basidiomycota and Neocallimastigomycota. Dominant classes were Dothideomycetes, Eurotiomycetes, Leotiomycetes, Saccharomycetes, Sordariomycetes (Ascomycota), Tremellomycetes, Wallemiomycetes (Basidiomycota) and Neocallimastigomycetes. Dominant orders were Capnodiales, Eurotiales, Pleosporales, Saccharomycetales, Thelebolales (Ascomycota), Filobasidiales (Basidiomycota) and Neocallimastigales. Dominant families were Aspergillaceae, Phaffomycetaceae, Sporormiaceae, Thelebolaceae (Ascomycota), Bulleribasidiaceae, Filobasidiaceae, Wallemiaceae (Basidomycota) and Neocallimastigaceae. Dominant genera were Aspergillus, Preussia, Thelebolus, Wickerhamomyces (Ascomycota), Naganishia, Vishniacozyma Wallemia (Basidiomycota) and Piromyces (Neocallimastigomycota). Dominant species were Aspergillus proliferans, Wickerhamomyces anomalus (Ascomycota) and Vishniacozyma victoriae, Wallemia muriae, W. sebi (Basidiomycota). 
Table 2 List of 56 key phylotypes that were associated with EGS

\begin{tabular}{|c|c|c|c|}
\hline Phylum & Class & Family & Phylotype \\
\hline \multirow[t]{21}{*}{ Ascomycota } & \multirow[t]{5}{*}{ Dothideomycetes } & Didymellaceae & Endophoma_elongata, Phomatodes_aubrietiae \\
\hline & & Didymosphaeriaceae & Paraconiothyrium_brasiliense \\
\hline & & Phaeosphaeriaceae & G_Phaeosphaeria \\
\hline & & Sporormiaceae & Preussia_tetramera, G_Preussia \\
\hline & & Unidentified & O_Pleosporales \\
\hline & \multirow[t]{3}{*}{ Eurotiomycetes } & Herpotrichiellaceae & Exophiala_pisciphila, F_Herpotrichiellaceae \\
\hline & & Trichocomaceae & Thermomyces_lanuginosus \\
\hline & & Unidentified & O_Onygenales \\
\hline & \multirow[t]{4}{*}{ Leotiomycetes } & Psendeurotiaceae & Pseudeurotium_bakeri, Pseudeurotium_ovale \\
\hline & & Thelebolaceae & Cleistothelebolus_nipigonensis \\
\hline & & & Thelebolus_globosus, Thelebolus_spongiae, G_Thelebolus \\
\hline & & Unidentified & O_Helotiales \\
\hline & Saccharomycetes & Unidentified & O_Saccharomycetales \\
\hline & \multirow[t]{8}{*}{ Sordariomycetes } & Unidentified & O_Coniochaetales \\
\hline & & Bionectriaceae & Gliomastix_tumulicola \\
\hline & & Hypocreaceae & Monocillium_griseo-ochraceum \\
\hline & & Hypocreales_fam_Incertae_sedis & Acremonium_rutilum, G_Acremonium \\
\hline & & Hypocreales_fam_Incertae_sedis & Chlamydocillium_cyanophilum \\
\hline & & Hypocreales_fam_Incertae_sedis & F_Hypocreales_fam_Incertae_sedis \\
\hline & & Lasiosphaeriaceae & F_Lasiosphaeriaceae \\
\hline & & Microascaceae & Kernia_retardata \\
\hline \multirow[t]{21}{*}{ Basidiomycota } & \multirow[t]{5}{*}{ Agaricomycetes } & Agaricaceae & Coprinus_cordisporus \\
\hline & & Entolomataceae & Entoloma_sericeum \\
\hline & & Psathyrellaceae & G_Coprinopsis \\
\hline & & unidentified & O_Agaricales \\
\hline & & unidentified & C_Agaricomycetes \\
\hline & \multirow[t]{3}{*}{ Cystobasidiomycetes } & Cystobasidiaceae & Cystobasidium_minuta, Cystobasidium_ritchiei \\
\hline & & Buckleyzymaceae & Buckleyzyma_aurantiaca \\
\hline & & unidentified & O_Erythrobasidiales \\
\hline & \multirow[t]{4}{*}{ Microbotryomycetes } & Chrysozymaceae & Bannozyma_yamatoana \\
\hline & & & F_Chrysozymaceae \\
\hline & & unidentified & O_Leucosporidiales \\
\hline & & unidentified & O_Sporidiobolales \\
\hline & \multirow[t]{7}{*}{ Tremellomycetes } & Bulleribasidiaceae & Vishniacozyma_foliicola \\
\hline & & Filobasidiaceae & Naganishia_adeliensis, Naganishia_albida \\
\hline & & Mrakiaceae & Tausonia_pullulans \\
\hline & & Piskurozymaceae & Solicoccozyma_phenolica \\
\hline & & Trimorphomycetaceae & Saitozyma_podzolica \\
\hline & & Trichosporonaceae & Apiotrichum_dulcitum \\
\hline & & unidentified & C_Tremellomycetes \\
\hline & unidentified & unidentified & P_Basidiomycota \\
\hline & Wallemiomycetes & Wallemiaceae & Wallemia_canadensis, Wallemia_sebi, Wallemia_tropicalis \\
\hline Chytridiomycota & unidentified & unidentified & P_Chytridiomycota \\
\hline Mortierellomycota & Mortierellomycetes & Mortierellaceae & Mortierella_gamsii \\
\hline unidentified & unidentified & unidentified & K_unidentified \\
\hline
\end{tabular}

Key taxa had significantly increased abundance in EGS samples, variable importance in projection scores $>1.5$, and both high abundance and high prevalence in EGS samples. $\mathrm{K}=$ kingdom, $\mathrm{P}=$ phylum, $\mathrm{C}=$ class, $\mathrm{O}=$ order, $\mathrm{F}=$ family, $\mathrm{G}=$ genus 
FUNGuild analysis parsed $87 \%$ of the 2816 phylotypes into 20 growth morphologies, 26 ecological guilds and 3 tropic modes. Most were classified as environmental microfungi, agaricoids or yeasts, comprising wood, soil, plant, dung and undefined saprotrophs, plant pathogens, endophytes, animal pathogens, fungal parasites and ectomycorrhizal fungi. These fungi are typical of those colonising grassland soils and plants [33] and show considerable overlap with those identified in a metagenomic study of grass endophytes [34].

The GI mycobiota of grazing horses appears to be richer and more diverse than that of humans and mice, which typically comprise nearly 70 genera and more than 184 species of fungi, but with 10 or fewer taxa typically accounting for the vast majority of fungi detected [3538]. The marked richness and diversity of the equine GI mycobiota likely reflects the richness and diversity of the environmental fungi present in the plants, soil and water that are ingested by grazing horses. Consistent with this, the diversity of fungal species in equine faeces was considered to reflect the different forage types fed to stabled horses [39]. Similarly, most of the fungi detected in human faeces are derived from the consumption of different foods, which contain, as a whole, more unique fungi than the population colonising the GI tract [40]. Many of the fungi commonly considered to represent the human core GI mycobiota, including Candida, Saccharomyces, Penicillium, Aspergillus, Cryptococcus, Malassezia, Cladosporium and Trichosporon [36, 41, 42], were detected in the equine GI tract. The predominant fungal phyla in both equine and human GI tracts are Ascomycota and Basidiomycota, while Neocallimastigomycota, GI adapted anaerobic fibre degrading endosymbionts, are abundant only in the horse [43, 44]. Neocallimastigomycota are an essential part of the core mycobiota colonising the equine GI tract, but the composition of the remainder of the equine core GI mycobiota is unknown and cannot be determined from this study alone. Indeed it is currently unclear whether the aerobic fungi detected in the GI tract of horses and other animals, including man, are true endosymbionts, opportunistic pathogens which colonise the GI tract only under particular circumstances, or are ingested fungi from food, water, environment, and nasal or oral cavities, and which are simply transiting through the GI tract [36, 42]. While the majority of aerobic fungi detected in the equine GI tract are likely to be transient non-colonising ingested environmental fungi, because these can maintain metabolic activity during GI transit [45], they could potentially contribute to EGS aetiology by producing extrolites in vivo. Opportunistic fungal pathogens which have colonised the equine GI tract could also contribute to EGS aetiology by producing toxic extrolites in vivo.
Mycobiota richness (Chao1) varied throughout the equine GI tract, being higher distally (caecum, colon, faeces) than proximally (stomach, ileum). PLS-DA and weighted UniFrac distance analysis (beta-diversity) identified significant differences in mycobiota structure among GI sites in both EGS and CTRL groups. Neocallimastigomycota, Neocallimastigomycetes, Neocallimastigales and Neocallimastigaceae were more abundant in distal than proximal GI sites, consistent with previous findings [46]. In contrast, Wallemiomycetes, Wallemiales, Wallemiaceae and Tremellales were more abundant proximally.

\section{EGS is associated with changes in the richness, diversity and structure of the GI mycobiota}

EGS is associated with significant alterations in the GI mycobiota. Mycobiota richness (Chao1) was higher in the caecum and colon of EGS horses compared with CTRL horses, while mycobiota diversity (Inverse Simpson) was higher in EGS colon and faeces, compared with CTRL and CoG horses, respectively. Indices of beta-diversity demonstrated inter-group differences in mycobiota structure at all taxonomic levels. Analysis with the Bioconductor software package DESEq2 identified a large number of phylotypes that were differentially abundant between EGS and the 2 control groups, most of which had increased abundance in EGS horses. PLSDA and VIP scores $(>1.5)$ were used to identify those taxa that were the most important contributors to the inter-group differential mycobiota structure. Key phylotypes $(n=56)$ associated with EGS, and which could have a potential role in EGS aetiology, were then identified which had a) high abundance and high prevalence in EGS samples, b) significantly increased abundance in EGS samples, and c) a VIP score $>1.5$ indicating they contributed significantly to inter-group differences in mycobiota structure. Key phylotypes comprised fungi of diverse taxonomy. FUNGuild analysis parsed the key phylotypes as predominantly environmental microfungi, classified as soil, dung, wood, plant and undefined saprotrophs, fungal parasites, plant pathogens, endophytes and animal pathogens. Some key phylotypes were macrofungi; Entoloma sericeum, G_Coprinopsis, O_Agaricales and C_Agaricomycetes.

The increased abundance of key phylotypes in the GI tract of EGS horses could reflect increased GI colonisation by opportunistic pathogenic fungi, but more likely reflects ingestion of increased numbers of these fungi in plants, litter and soil while grazing. Indeed there is evidence to suggest that EGS horses are exposed to increased numbers of a wide range of diverse environmental microbes. In addition to key fungi, EGS horses had increased abundance of K_Rhizaria (G_Cercozoa), 
heterotrophic protists that predate bacteria thereby substantially changing the structure and function of microbial communities on plant surfaces [47-49]. Further, a previous study identified increased numbers of cyanobacteria, filamentous green algae, unicellular green algae, diatoms, motile algal flagellates and desmids (Closterium sp.) on plants growing on EGS pastures during disease outbreaks [50]. It is likely that this increased abundance of a wide range of diverse microbes on EGS fields reflects favourable environmental conditions for microbial growth, including suitable vegetation, soil organic matter content, $\mathrm{pH}$, conductivity, temperature and availability of water and macronutrients [51]. Conditions which favour fungal growth and/or extrolite elaboration on the pasture could potentially account for some of the environmental risk factors for EGS. These factors include spring and early summer season, cool, dry weather with irregular ground frosts, faecal contamination, sand and loam rather than chalk soils, high soil nitrogen and low $\mathrm{Cu}$ and $\mathrm{Zn}$, and pasture disturbance $[15,20,52]$. Many key phylotypes are extremophilic fungi, including Pseudeurotium, Thelobolus (psychrophilic), Thermomyces lanuginosus (thermophilic), Wallemia (xerophilic) and extremophilic or polyextremophilic yeasts including Apiotrichum, Bannozyma, Cystobasidium, Nagashinia, O_Saccharomycetales, Saitozyma, Tausonia and Vishniacozyma [51, 53, 54]. Increased abundance of these extremophiles likely reflects their ability to survive the potentially adverse environmental conditions associated with EGS, including cold and dry weather. The increased abundance of some soil yeasts may also be attributable to extracellular polysaccharide capsules that facilitate survival in sandy soils [51] which are associated with EGS [20]. Many key phylotypes are dung saprotrophs, including Acremonium spp. O_Agaricales, Coprinus spp., Coprinopsis spp., Pleospora spp., Preussia spp., Thelebolus spp. [55], Cleistothelebolus nipigonensis, O_Coniochaetales and Kernia retardata, potentially explaining the reduction in EGS risk when faeces are collected manually from pastures [20].

Alternatively, the alterations in mycobiota associated with EGS could be a consequence, rather than a cause of EGS, perhaps attributable to the GI stasis which characterises the disease. Consistent with this possibility, experimental murine antimycotic drug-induced intestinal fungal dysbiosis resulted in increased abundance of Wallemia [56], one of the key phylotypes associated with EGS. Further work is therefore required to determine whether any of the key phylotypes contribute to EGS aetiology or whether their association with EGS is a consequence of the disease. None of the key phylotypes has been previously associated with a pasture neuromycotoxicosis resembling
EGS, however many of them are predicted to produce cytotoxic and/or neurotoxic extrolites, including brefeldin, communiols, cytochalasans, d-lysergic acid amide, gliotoxin, L-DOPA, polyketides, preussins, sesquiterpenoids, trichothecenes, tyrosinase and walleminol [39, 57-59]. In addition, antibacterial and antifungal activities of fungal extrolites within the GI tract [60] could potentially induce the marked GI bacterial dysbiosis which occurs in EGS [61] and contribute to the changes in mycobiota reported herein. Examination of GI contents from EGS horses for those extrolites produced by key phylotypes is therefore warranted to further test the hypothesis that they contribute to EGS aetiology.

It is conceivable that EGS is more prevalent in horses when there is a reduced abundance of particular taxa that serve key beneficial functions for the host. Notable taxa with reduced abundance in EGS horses included some species of Alternaria and Cladosporium, dominant endophytic fungi on temperate grasses [62], Fusarium, an animal and plant pathogen, soil and wood saprotroph, endophyte, and lichen parasite, Neoascochyta, an animal and plant pathogen and saprotroph, and the plant pathogen Mycosphaerella tassiana. Consistent with these findings, Doxey et al. [22] isolated Fusarium and Alternaria only infrequently from EGS horses. In contrast, Robb et al. [24] identified Fusarium on plants from all EGS fields in Scotland and Patagonia. Rather than contributing to EGS aetiology, reduced numbers of these aforementioned taxa likely reflects reductions in the numbers of these fungi that are ingested by EGS horses, perhaps because the environmental conditions associated with EGS are unfavourable for growth of these fungi. Alternatively, reduced numbers of certain taxa may be attributable to the inhibitory effects of antifungal extrolites produced by those taxa that were present in increased abundance.

The structure of the faecal mycobiota of CoG horses differed significantly from those of EGS and CTRL horses. CoG horses were co-grazing with EGS horses at the time of disease onset. While CoG horses remain clinically healthy, as with EGS horses, they have increased serum concentrations of acute phase proteins [32] consistent with subclinical exposure to the toxin that causes EGS. It is possible that the quantity of causal fungi/extrolite ingested by CoG horses is sufficient to induce a self-resolving acute inflammatory response but insufficient to induce clinical EGS. Consistent with this possibility, 29 of the 56 key phylotypes associated with EGS were more abundant in faeces of EGS versus CoG groups. Alternatively CoG horses may survive exposure to the causal toxin because of hostspecific immunological protection or toxin metabolism. 


\section{Study limitations}

Targeted amplicon sequencing identified considerably many more fungi than a previous culture-based study of the GI mycobiota of EGS and control horses [22]. However, this methodology has biases and limitations [43], including underestimation, or failure to detect, taxa lacking validated phylogenetic marker sequences, such as some grass endophytes [62] and Fusarium [63]. This may also explain why several taxa isolated from the equine GI tract by Doxey et al. [22] were not identified in the present study, including Absidia, Rhizopus, Thamnidium, Geotrichum (Dipodascus) and Monilia. The limited annotation of some fungal genes can also preclude classification of taxa to fine taxonomic ranks. In the present study, most taxa were identified to genus level, but only $40.1 \%$ were identified to species level, and some taxa, including key taxa, were identified only at kingdom or phylum levels.

While all of the fungi potentially present in the equine GI tract of grazing horses will not have been identified in this study, this was not considered to be a significant limitation given that the main aim of the study was to identify key taxa associated with EGS, rather than to generate an inventory of the equine GI mycobiota.

Inclusion of mock community and negative controls is considered an essential feature of amplicon sequencing experiments, to identify biases and sample contamination, respectively [63]. All 12 taxa in the two mock communities were identified in all mock community samples. Samples of mock community 1 had markedly lower amplicon numbers for Saccharomyces cerevisiae than for Cryptococcus neoformans. Similarly, Bakker [63] failed to detect $S$. cerevisiae in an ITS1 amplicon library, concluding that this negative bias was likely associated with amplicon length. We have noted that the number and depth of OTUs is immensely sensitive to the stringency of the parameters applied during assembly (data not shown), and thus the abundance of rRNA gene amplicons may not accurately reflect taxon biomass in samples [63]. Some taxa, including Coniochaeta lignicola which was included in mock community 2, were represented by a single OTU. Others, including Vishniacozyma victoriae, were represented by multiple OTUs, reflecting intragenomic marker gene polymorphism [64]. To mitigate against these observations, a phylotype approach, rather than an OTU approach, was adopted for this study [63].

Negative control samples are valuable for revealing rDNA contamination, but there is little or no consensus regarding how to incorporate information from negative control samples into data processing [63]. All negative control samples had low amplicon counts, except 3 which were contaminated with rDNA from Alternaria infectoria and Mycosphaerella tassiana. As these two taxa were included in mock community 2 , and were present in many GI samples, it was considered inappropriate to remove these taxa from the GI sample analysis [63]. Importantly, these contaminant taxa were not key taxa and were not increased in abundance in EGS samples. Retrospective analysis indicated that 53 of the 13,204 OTUs were likely contaminants; none of these contributed to the key phylotypes.

EGS horses were significantly younger than controls, in part reflecting the difficulty obtaining post-mortem samples from young control horses. While age influences mycobiota structure [65], it seems unlikely to account for the significant inter-group differences observed herein, which are instead consistent with inter-group differences in exposure to a diverse range of environmental fungi.

\section{Conclusions}

The equine GI mycobiota comprises a very large and diverse population of fungi, varying in growth form, trophic mode and ecological guild. Most are ingested environmental fungi, probably in transit through the GI tract. EGS horses had a significantly richer, more diverse, and structurally different, GI mycobiota than two control populations. A large number of taxa showed statistically significant differential abundances between groups. Key phylotypes $(n=56)$ associated with EGS were identified, many of which are extremophiles capable of producing cytotoxic and/or neurotoxic extrolites. Further work is required to determine whether extrolites produced by key phylotypes contribute to EGS aetiology or whether the association of key phylotypes and EGS is non-causal or is a consequence of the disease.

\section{Methods}

\section{Sample collection and processing}

GI contents were collected post mortem, typically within $4 \mathrm{~h}$ of euthanasia on humane grounds by barbiturate overdose, from up to 5 sites, namely stomach, ileum, caecum, colon and rectum (faeces) of EGS horses (150 samples from 54 horses) and from control grazing horses that had been euthanased on humane grounds for reasons other than neurologic or GI diseases (CTRL group; 67 samples from 31 horses) (Additional file 5). We were unable to sample all GI sites in all horses. EGS horses comprised 39 acute EGS and 15 sub-acute EGS horses, categorised as previously described [25], and confirmed by histopathology of autonomic ganglia. Freshly voided faecal samples were also collected from 48 healthy horses that were co-grazing (CoG group) with the EGS horses when the latter developed EGS, typically within $48 \mathrm{~h}$ of disease onset. Samples were promptly frozen at -20 or $-80{ }^{\circ} \mathrm{C}$. In addition to the 265 GI samples described, 
an additional 26 samples were collected and processed, but were not included in the subsequent analyses because they generated too few $(<5000)$ amplicon sequences.

Negative (nuclease-free water) and positive mock fungal community controls were run in parallel with samples. Initial samples were run in parallel with mock community 1 (10 ng DNA per sample), a mixed microbial population which included rDNA from Saccharomyces cerevisiae and Cryptococcus neoformans (ZymoBIOMICS Microbial Community DNA Standard, Zymo Research, Irvine, USA). Subsequent samples were run with mock community 2 , which was prepared to provide a community of 10 fungi (Alternaria infectoria, Coniochaeta lignicola, Didymella rumicicola, Mycosphaerella tassiana, Penicillium pagulum, Pyrenochaetopsis pratorum, Vishniacozyma victoriae, Xylaria longipes, G_Eutypella and G_Fusarium) which colonised grasses collected from equine pastures within the geographical area from which the equine GI samples were collected. To prepare mock community 2, plant stems and crowns were surface sterilised [66] and cultured on corn meal agar at room temperature. On day 10, 33 isolates were selected and sub-cultured and then curated on day 28. Mycelia were harvested from 10 selected isolates, homogenised mechanically and rDNA extracted using a standard CTAB DNA extraction protocol. DNA was quantified using NanoDrop 2000 spectrophotometer (NanoDrop Technologies, Wilmington, DE, USA), and quantity assessed using Qubit 3 (Invitrogen, Inchinnan, UK). Mock community 2A contained $5 \mathrm{ng}$ DNA from all 10 isolates, while mock community $2 \mathrm{~B}$ contained $50 \mathrm{ng}$ DNA of Didymella rumicicola and Xylaria longipes and $5 \mathrm{ng}$ for other isolates.

For GI samples, total DNA was extracted with the AllPrep PowerFecal DNA/RNA Kit (QIAGEN, Hilden, Germany), following manufacturer's instructions. A bead beating step was included (FastPrep-24 5G bead beating grinder and lysis system, MP Biomedicals, Eschwege, Germany). The purity of the resulting DNA extract was assessed using NanoDrop 2000 spectrophotometer (NanoDrop Technologies, Wilmington, DE, USA), and the quantity assessed using Qubit 3 (Invitrogen, Inchinnan, UK) and the Agilent High Sensitivity D1000 ScreenTape System (2200)(Agilent Technologies, Santa Clara, USA). Pooled libraries also underwent quality control checks by Edinburgh Genomics [67] prior to sequencing using the High Sensitivity D1K ScreenTape (Agilent Technologies, Santa Clara, USA).

The Internal Transcribed Spacer 1 (ITS1) region was amplified using primers ITS1F (FWD 5`-CTTGGTCAT TTAGAGGAAGTAA-3`) and ITS2 (REV 5`- GCTGCG TTCTTCATCGATGC -3`) [68], using 50 ng DNA per sample. Amplification was performed on a Mycycler
Thermal Cycler (Bio-Rad, Watford, UK). PCR cycle conditions, selected based on primer recommendations, were $95{ }^{\circ} \mathrm{C}$ for $2 \mathrm{~min}, 95^{\circ} \mathrm{C}$ for $30 \mathrm{~s}, 55^{\circ} \mathrm{C}$ for $30 \mathrm{~s}$ and $72{ }^{\circ} \mathrm{C}$ for $1 \mathrm{~min}(\times 30$ cycles $)$ and $72{ }^{\circ} \mathrm{C}$ for $7 \mathrm{~min}$. DNA amplicons were purified using the AMPure XP PCR Purification System (Beckman Coulter, High Wycombe, UK) according to manufacturer's instructions. Amplicons were paired-end $(2 \times 250 \mathrm{nt})$ sequenced on Illumina MISeq v2 platform (Illumina, San Diego, USA).

\section{Bioinformatics and statistical analyses}

Microbial composition summary box plots, alpha diversity, weighted UniFrac based Partial Least-Squares Discriminant Analysis (PLS-DA) were performed within $\mathrm{R}$ version 3.6.0 (2019-04-26) [69] using the following packages: phyloseq [70], metagenomeSeq [71], vegan [72], ape [73], ggplot2 [74], mixOmics [75], DESeq2 [76], GUniFrac [77] and dplyr [78]. A paired t-test on the sample count for each month was used to determine whether there was an inter-group difference in month of collection of EGS and CTRL samples. Chi squared test was used to determine whether there were inter-group differences in horse sex. Mann Whitney tests were used to determine whether there were inter-group differences in horse age.

Raw sequence data from 3 MiSeq runs were pooled and analysed with PIPITS [79], an open-source (https:// sourceforge.net/projects/pipits) stand-alone suite of software for automated processing of Illumina MiSeq sequences for fungal community analysis, incorporating ITSx to extract subregions of ITS and exploiting the latest RDP Classifier to classify sequences against the curated UNITE fungal ITS reference data set [80]. GI samples ( $n=26$ from all groups) with total amplicon read counts $<5000$ were not included in the study, leaving 265 GI samples for analysis. Sequencing depth was assessed using rarefaction curves. Taxonomy plots were constructed for phylum, class, order and family, using data normalised to relative abundance. To aid visualisation, plots were constructed using data filtered at $0.05 \%$ abundance. Data were analysed using the $\mathrm{R}$ package decontam [81]; since this was done retrospectively, identified contaminants were not removed and were included in the analyses.

FUNGuild [33] was used to taxonomically parse phylotypes to defined ecological guilds, trophic modes and growth morphologies. Alpha-diversity was measured using Chao1 (richness estimator that accounts for sequencing depth) and Inverted Simpson (diversity). Alpha-diversity indices were calculated at phylotype level using the "Phyloseq" R package [70]. Kruskal-Wallis rank sum test and Wilcoxon rank sum test with continuity 
correction were used to determine whether indices differed among GI sites and among groups.

Partial least squares discriminant analysis (PLS-DA), a supervised model based on a least squares regression model, was used to reveal inter-group and inter-site variation in mycobiota structure at phylotype level. Differences were assessed by performing an adonis analysis based on weighted UniFrac distances, a phylogenetic based distance metric which, when weighted, accounts for the relative abundance of phylotypes [82]. DESeq2 analysis was used for inter-group comparisons (EGS versus CTRL, EGS versus CoG and CoG versus CTRL) of mycobiota, for each GI site separately, at all taxonomic levels except kingdom, at phylotype level. PLS-DA and variable importance in projection (VIP) scores were used to identify phylotypes that were important contributors to the differential mycobiota structure in EGS versus CTRL groups, EGS versus CoG groups and CoG versus CTRL groups, using the "plsda" function in the $\mathrm{R}$ package "mix Omics" $[75,83]$. Phylotypes with VIP scores $>1.5$ were considered to be important contributors to the model [84]. Key phylotypes associated with EGS, and which could have a potential role in EGS aetiology, were identified that had; a) high abundance in EGS samples ie being within the top 50\% quantile of abundant phylotypes, b) high prevalence in EGS samples ie present in $>50 \%$ of EGS samples included in each paired comparison, c) a VIP score $>1.5$ and significantly increased abundance $[\mathrm{Padj}<0.05, \log 2$ fold change $>0$ ] in EGS samples in any comparison of EGS versus CTRL (overall, stomach, ileum, caecum, colon, faeces) or EGS versus CoG (faeces) samples. For this, DESeq2 and PLS-DA/VIP analyses were performed including only those phylotypes which had the aforementioned high prevalence and abundance in EGS samples.

\begin{abstract}
Abbreviations
CoG: Co-grazing group; CTRL: Control group; EGS: Equine grass sickness; Gl: Gastrointestinal; ITS: Internal transcribed spacer; OTU: Operational taxonomic unit; PCOA: Principal coordinate analysis; PCR: Polymerase chain reaction;" PLS-DA: Partial least squares discriminant analysis; VIP: Variable importance in projection.
\end{abstract}

\section{Supplementary Information}

The online version contains supplementary material available at https://doi. org/10.1186/s42523-021-00131-2.

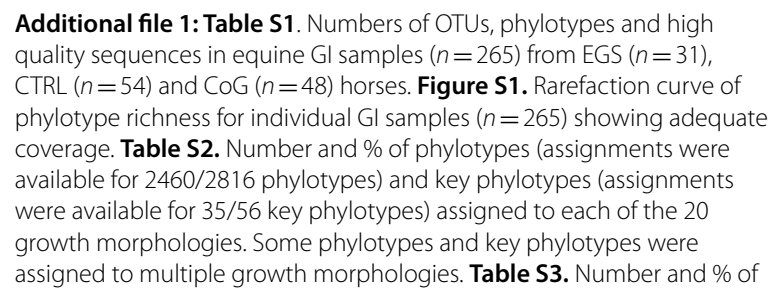

phylotypes (assignments were available for 2460/2816 phylotypes) and key phylotypes (assignments were available for 35/56 key phylotypes) assigned to each of the 3 FUNGuild trophic modes. Some phylotypes and key phylotypes were assigned to multiple trophic modes. Table S4. Number and \% of phylotypes (assignments were available for 2460/2816 phylotypes) and key phylotypes (assignments were available for 35/56 key phylotypes) assigned to each of 26 ecological guilds. Some phylotypes and key phylotypes were assigned to multiple ecological guilds. Figure S2. Taxonomy plots showing relative abundance of taxa at (A) order and (B) family levels. Data are filtered at $0.05 \%$ abundance threshold. Table S5. Statistical comparison of indices of alpha-diversity across all 5 GI sites (overall) and between paired GI sites. Data pooled for all horses; ANOVA, P values. Significant differences in bold, letter indicates higher value $(\mathrm{Ca}=$ caecum; $\mathrm{F}=$ faeces $)$. Table $\mathbf{S 6}$. Weighted UniFrac distance analysis identified significant inter-site dissimilarity in mycobiota structure in EGS and CTRL horses, at phylotype level. P values, statistically different comparisons are indicated in bold. Table S7. Inter-group weighted UniFrac distance analysis at different GI sites, at phylotype level (P values). Statistically significant dissimilarity is indicated in bold. Table S8. Numbers of differentially abundant taxa, at phylum, class, order, family, genus and phylotype levels.

Additional file 2. Differentially abundant (increased and decreased) taxa. Separate sheets show differentially abundant taxa at Phylum, Class, Order, Family, Genus, phylotype (increased and decreased abundance) and OTU (increased and decreased abundance) levels. Separate columns show statistical data for comparisons overall (OV; pooled data for all Gl sites) and at paired GI sites (ST; stomach, IL; ileum, CA; caecum, CO; colon, FA; faeces), for EGS vs CTRL, and for faeces for EGS vs CoG and for CoG vs CTRL groups. Separate columns show adjusted $p$ value (padj) and $\log _{2}$ fold change, and for phylotype data, VIP scores for statistically significant paired comparisons.

Additional file 3. List of key phylotypes associated with EGS $(n=56)$. Separate columns show taxonomy of key phylotypes and statistical data for comparisons overall (OV; pooled data for all Gl sites) and at paired Gl sites (ST; stomach, IL; ileum, CA; caecum, CO; colon, FA; faeces), for EGS vs CTRL, and for faeces for EGS vs CoG and for CoG vs CTRL groups. Separate columns show adjusted p value (padj) and $\log _{2}$ fold change, and for phylotype data, VIP scores for statistically significant paired comparisons.

Additional file 4. FUNGuild parsing of key phylotypes. Separate columns show taxonomy of key phylotypes and their FUNGuild identity, taxon level, trophic mode, guild, growth form, trait, notes, confidence rank, and citation/source, as described by Nguyen et al. (2016) [33] and https:// github.com/UMNFuN/FUNGuild.

Additional file 5. Horse metadata. Separate columns show sample number, case identity, group, category, GI site, collection date, month of sample collection, premises code (anonymised), age (years), sex and breed. AGS acute grass sickness; SAGS sub-acute grass sickness; ID Irish Draught; TB Thoroughbred; WB Warmblood; x crossbred.

\section{Acknowledgements}

We thank Gavin Paterson, Jennifer Harris and Laura Hamilton for assistance preparing mock community DNA.

\section{Authors' contributions}

B.M, G.M., S.P. and J.T. designed the study; B.M., S.P., J.K. and L.H. collected GI samples; L.H. and T.W. prepared libraries; J.T. prepared mock community DNA; H.G. performed PIPITS analysis; B.M., L.H., Z.C. and A.I. analysed data; B.M., G.M., L.G. and J.T. interpreted the results and wrote the manuscript. All authors read and approved the final manuscript.

\section{Funding}

This study was funded by The Horse Trust (horsetrust.org.uk). Collection of some archived samples was funded by The Equine Grass Sickness Fund (grasssickness.org.uk). 


\section{Availability of data and materials}

Sequencing reads can be accessed in the European Nucleotide Archive under accession number PRJEB45209.

\section{Declarations}

\section{Ethics approval and consent to participate}

The study was approved by the Royal (Dick) School of Veterinary Medicine Veterinary Ethical Review Committee, University of Edinburgh (reference number 60.21).

\section{Consent for publication}

Not applicable.

\section{Competing interests}

The authors declare no competing interests.

\section{Author details}

${ }^{1}$ Royal (Dick) School of Veterinary Studies and The Roslin Institute, Easter Bush Veterinary Centre, University of Edinburgh, Roslin, Midlothian EH25 9RG, UK.

${ }^{2}$ Ashworth Laboratories, University of Edinburgh, Edinburgh EH9 3FL, UK.

${ }^{3}$ School of Biological Sciences, University of Reading, Reading RG6 6EX, UK.

${ }^{4}$ Royal Botanic Garden Edinburgh, 20A Inverleith Row, Edinburgh EH3 5LR, UK.

Received: 9 June 2021 Accepted: 14 September 2021

Published online: 09 October 2021

\section{References}

1. Pirie RS, McGorum BC. Equine grass sickness: an update. UK Vet. 2018. https://doi.org/10.12968/ukve.2018.2.1.6.

2. Pirie RS, Jago RC, Hudson NPH. Equine grass sickness. Equine Vet J. 2014;46:545-53. https://doi.org/10.1111/evj.12254.

3. Pollin MM, Griffiths IR. A review of the primary dysautonomias of domestic animals. J Comp Path. 1992;106:99-119. https://doi.org/10.1016/00219975(92)90041-R

4. Hahn CN, Mayhew IG, de Lahunta A. Central neuropathology of equine grass sickness. Acta Neuropathol. 2001;102(2):153-9. https://doi.org/10 1007/s004010000289.

5. Sharp NJH, Nash AS, Griffiths IR. Feline dysautonomia (the Key-Gaskell syndrome): a clinical and pathological study of forty cases. J Small An Prac. 1984;25:599-615. https://doi.org/10.1111/j.1748-5827.1984.tb033 72.x.

6. Whitwell KE. Do hares suffer from grass sickness? Vet Rec. 1991:128(17):395-6. https://doi.org/10.1136/vr.128.17.395.

7. Longshore RC, O'Brien DP, Johnson GC, Grooters AM, Kroll RA. Dysautonomia in dogs: a retrospective study. J Vet Intern Med. 1996;10(3):103-9. https://doi.org/10.1111/j.1939-1676.1996.tb02040.x.

8. Kik MJ, van der Hage MH. Cecal impaction due to dysautonomia in a Ilama (Lama glama). J Zoo Wildl Med. 1999;30(3):435-8.

9. Pruden SJ, MCAllister MM, Schultheiss PC, O'Toole D, Christensen DE. Abomasal emptying defect of sheep may be an acquired form of dysautonomia. Vet Pathol. 2004;41(2):164-9. https://doi.org/10.1354/vp. 41-2-164.

10. Hahn CN, Whitwell KE, Mayhew IG. Neuropathological lesions resembling equine grass sickness in rabbits. Vet Rec. 2005;156:778-9. https://doi.org/ 10.1136/vr.156.24.778

11. Lewis CA, Bozynski CC, Johnson GC, Harral CM, Williams F 3rd, Tyler JW. Colonic impaction due to dysautonomia in an alpaca. J Vet Intern Med. 2009;23(5):1117-22. https://doi.org/10.1111/j.1939-1676.2009.0351.x.

12. Hunter LC, Miller JK, Poxton IR. The association of Clostridium botulinum type $C$ with equine grass sickness: A toxicoinfection? Equine Vet J. 1999;31(6):492-9. https://doi.org/10.1111/j.2042-3306.1999.tb03857.x.

13. Hunter LC, Poxton IR. Systemic antibodies to Clostridium botulinum type C: Do they protect horses from grass sickness (dysautonomia)? Equine Vet J. 2001;33(6):547-53. https://doi.org/10.2746/042516401776563418.

14. McCarthy HE, French NP, Edwards GB, Poxton IR, Kelly DF, Payne-Johnson CE, Miller K, Proudman CJ. Equine grass sickness is associated with low antibody levels to Clostridium botulinum: a matched case-control study.
Equine Vet J. 2004;36(2):123-9. https://doi.org/10.2746/0425164017 76563418.

15. Doxey DL, Gilmour JS, Milne EM. The relationship between meteorological features and equine grass sickness (dysautonomia). Equine Vet J. 1991;23(5):370-3. https://doi.org/10.1111/j.2042-3306.1991.tb03740.x.

16. Wambacq E, Vanhoutte I, Audenaert K, De Gelder L, Haesaert G. Occurrence, prevention and remediation of toxigenic fungi and mycotoxins in silage: a review. J Sci Food Agric. 2016;96(7):2284-302. https://doi.org/10. 1002/jsfa.7565.

17. Wood JLN, Milne EM, Doxey DL. A case-control study of grass sickness (equine dysautonomia) in the United Kingdom. Vet J. 1998;156(1):7-14. https://doi.org/10.1016/S1090-0233(98)80055-5.

18. Wylie CE, Shaw DJ, Fordyce FM, Lilly A, McGorum BC. Equine grass sickness in Scotland: a case-control study of signalment- and meteorologyrelated risk factors. Equine Vet J. 2014;46(1):64-71. https://doi.org/10. 1111/evj.12077.

19. Doxey DL, Gilmour JS, Milne EM. A comparative study of normal equine populations and those with grass sickness (dysautonomia) in eastern Scotland. Equine Vet J. 1991;23(5):365-9. https://doi.org/10.1111/j.20423306.1991.tb03739.x

20. Newton JR, Hedderson EJ, Adams VJ, McGorum BC, Proudman CJ, Wood $J$ L. An epidemiological study of risk factors associated with the recurrence of equine grass sickness (dysautonomia) on previously affected premises. Equine Vet J. 2004;36(2):105-12. https://doi.org/10.2746/04251 64044868639.

21. McCarthy HE, Proudman CJ, French NP. Epidemiology of equine grass sickness: a literature review (1909-1999). Vet Rec. 2001;149(10):293-300. https://doi.org/10.1136/vr.149.10.293.

22. Doxey DL, Robb J, Milne EM, Gilmour JS. Mycological studies on the equine intestinal tract with particular reference to equine dysautonomia (grass sickness). Ann Appl Biol. 1990;117:337-41. https://doi.org/10. 1111/j.1744-7348.1990.tb04219.x.

23. di Menna ME, Waller JE. Visual assessment of seasonal changes in amount of mycelium of Acremonium loliae in leaf sheaths of perennial ryegrass. $N$ Z J Agric Res. 1986;29(1):111-6. https://doi.org/10.1080/00288233.1986. 10417982.

24. Robb J, Doxey D, Milne E, Whitwell K, Robles C, Uzal F, John H. The isolation of potentially toxigenic fungi from the environment of horses with grass sickness and mal seco. In: Hahn C, Gerber V, Mayhew IG editors. Proceedings of the 1st International Workshop on Grass Sickness, equine motor disease and related disorders. Newmarket: Equine Veterinary Journal; 1997. p. 52-4.

25. McGorum BC, Kirk J. Equine dysautonomia (grass sickness) is associated with altered plasma amino acid levels and depletion of plasma sulphur amino acids. Equine Vet J. 2001;33(5):473-7. https://doi.org/10.2746/ 042516401776254763.

26. McGorum BC, Symonds HW, Knottenbelt C, Cave TA, MacDonald SJ, Stratton J, Leon I, Turner JA, Pirie RS. Alterations in amino acid status in cats with feline dysautonomia. PLoS ONE. 2017;12(3): e0174346. https://doi. org/10.1371/journal.pone.0174346.

27. Veltmann JR, Wyatt RD, Voight MN, Shamsuddin Z. Influence of dietary sulphur amino acid levels on performance, free amino acids and biochemical parameters in plasma and hepatic glutathione of broiler chicks fed aflotoxin. Poultry Sci. 1983;62:1518-9.

28. Nahm KH. A study on the detoxification in the chick's body of aflatoxin B1 found in feed. Poulty Sci. 1991;70S:501.

29. Greig JR. Grass sickness in horses. Trans R Highl Agric Soc. 1942;54:1-27.

30. Uzal FA, Woodman MP, Giraudo CG, Robles CA, Doxey DL. An attempt to reproduce'mal seco' in horses by feeding them Festuca argentina. Vet Rec. 1996;139(3):68-70. https://doi.org/10.1136/vr.139.3.68.

31. Espersen G, Lorck H. Grass sickness in horses, ergot and ergotism. Kobenhaven Kongelige Veterinaer-og Landbohoejskole. Copenhagen: Institut for Kirurgi; 1988.

32. Copas VE, Durham AE, Stratford CH, McGorum BC, Waggett B, Pirie RS. In equine grass sickness, serum amyloid $A$ and fibrinogen are elevated, and can aid differential diagnosis from non-inflammatory causes of colic. Vet Rec. 2013;172(15):395. https://doi.org/10.1136/vr.101224.

33. Nguyen NH, Song Z, Bates ST, Branco S, Tedersoo L, Menke J, Schilling JS, Kennedy PG. FUNGuild: an open annotation tool for parsing fungal community datasets by ecological guild. Fungal Ecol. 2016;20:241-8. https:// doi.org/10.1016/j.funeco.2015.06.006. 
34. Wemheuer B, Thomas T, Wemheuer F. Fungal endophyte communities of three agricultural important grass species differ in their response towards management regimes. Microorganisms. 2019;7(2):37. https://doi.org/10. 3390/microorganisms7020037.

35. Zhang D, Wang Y, Shen S, Hou Y, Chen Y, Wang T. The mycobiota of the human body: a spark can start a prairie fire. Gut Microbes 2020;11(4):655-79. https://doi.org/10.1080/19490976.2020.1731287.

36. Hallen-Adams HE, Suhr MJ. Fungi in the healthy human gastrointestinal tract. Virulence. 2017;8(3):352-8. https://doi.org/10.1080/21505594.2016. 1247140

37. Mukherjee PK, Sendid B, Hoarau G, Colombel JF, Poulain D, Ghannoum MA. Mycobiota in gastrointestinal diseases. Nat Rev Gastroenterol Hepatol. 2015;12(2):77-87. https://doi.org/10.1038/nrgastro.2014.188.

38. Forbes JD, Bernstein CN, Tremlett H, Van Domselaar G, Knox NC. A fungal world: Could the gut mycobiome be involved in neurological disease? Front Microbiol. 2018;9:3249. https://doi.org/10.3389/fmicb.2018.03249.

39. Andersen B, Phippen C, Frisvad JC, Emery S, Eustace RA. Fungal and chemical diversity in hay and wrapped haylage for equine feed. Mycotoxin Res. 2020;36(2):159-72. https://doi.org/10.1007/ s12550-019-00377-5.

40. Auchtung TA, Fofanova TY, Stewart CJ, Nash AK, Wong MC, Gesell JR, Auchtung JM, Ajami NJ, Petrosino JF. Investigating colonization of the healthy adult gastrointestinal tract by fungi. mSphere. 2018;3(2):e00092 18. https://doi.org/10.1128/mSphere.00092-18.

41. Suhr MJ, Hallen-Adams HE. The human gut mycobiome: pitfalls and potentials - a mycologist's perspective. Mycologia. 2015;107(6):1057-73. https://doi.org/10.3852/15-147.

42. Fiers WD, Gao IH, Iliev ID. Gut mycobiota under scrutiny: Fungal symbionts or environmental transients? Curr Opin Microbiol. 2019;50:79-86. https://doi.org/10.1016/j.mib.2019.09.010.

43. Richard ML, Sokol H. The gut mycobiota: insights into analysis, environmental interactions and role in gastrointestinal diseases. Nat Rev Gastroenterol Hepatol. 2019;16(6):331-45. https://doi.org/10.1038/ s41575-019-0121-2.

44. García-Gamboa R, Kirchmayr MR, Gradilla-Hernández MS, Pérez-Brocal V, Moya A, González-Avila M. The intestinal mycobiota and its relationship with overweight, obesity and nutritional aspects. J Hum Nutr Diet. 2021 https://doi.org/10.1111/jhn.12864.

45. David LA, Maurice CF, Carmody RN, Gootenberg DB, Button JE, Wolfe BE, Ling AV, Devlin AS, Varma Y, Fischbach MA, Biddinger SB, Dutton RJ, Turnbaugh PJ. Diet rapidly and reproducibly alters the human gut microbiome. Nature. 2014;505(7484):559-63. https://doi.org/10.1038/natur e12820.

46. Mura E, Edwards J, Kittelmann S, Kaerger K, Voigt K, Mrázek J, Moniello G, Fliegerova K. Anaerobic fungal communities differ along the horse digestive tract. Fungal Biol. 2019;123(3):240-6. https://doi.org/10.1016/j.funbio. 2018.12.004.

47. Bonkowski M. Protozoa and plant growth: the microbial loop in soil revisited. New Phytol. 2004;162(3):617-31. https://doi.org/10.1111/j.14698137.2004.01066.x.

48. Xiong W, Jousset A, Guo S, Karlsson I, Zhao Q, Wu H, Kowalchuk GA, Shen Q, Li R, Geisen S. Soil protist communities form a dynamic hub in the soil microbiome. ISME J. 2018;12(2):634-8. https://doi.org/10.1038/ismej. 2017.171.

49. Flues S, Bass D, Bonkowski M. Grazing of leaf-associated Cercomonads (Protists: Rhizaria: Cercozoa) structures bacterial community composition and function. Environ Microbiol. 2017;19(8):3297-309. https://doi.org/10. 1111/1462-2920.13824

50. McGorum BC, Pirie RS, Glendinning L, McLachlan G, Metcalf JS, Banack SA, Cox PA, Codd GA. Grazing livestock are exposed to terrestrial cyanobacteria. Vet Res. 2015;46:16. https://doi.org/10.1186/s13567-015-0143-x.

51. Yurkov AM. Yeasts of the soil-obscure but precious. Yeast. 2018;35(5):369-78. https://doi.org/10.1002/yea.3310.

52. Wylie CE, Shaw DJ, Fordyce FM, Lilly A, Pirie RS, McGorum BC. Equine grass sickness in Scotland: a case-control study of environmental geochemical risk factors. Equine Vet J. 2016;48(6):779-85. https://doi.org/10. 1111/evj.12490

53. Buzzini P, Turk M, Perini L, Turchetti B, Gunde-Cimerman N. Yeasts in polar and subpolar habitats. In: Buzzini P, Lachance MA, Yurkov A, editors. Yeasts in natural ecosystems: diversity. Berlin: Springer; 2017. p. 331-65. https:// doi.org/10.1007/978-3-319-62683-3_11.

54. Wicklow D, Malloch D. Studies in the genus Thelebolus: temperature optima for growth and ascocarp development. Mycologia. 1971;63(1):118-31. https://doi.org/10.2307/3757693.

55. Piontelli E, Toro Santa-Maria MA, Caretta G. Coprophilous fungi of the horse. Mycopathologia. 1981;74:89-105. https://doi.org/10.1007/BF012 59464.

56. Wheeler ML, Limon JJ, Bar AS, Leal CA, Gargus M, Tang J, Brown J, Funari VA, Wang HL, Crother TR, Arditi M, Underhill DM, Iliev ID. Immunological consequences of intestinal fungal dysbiosis. Cell Host Microbe. 2016;19(6):865-73. https://doi.org/10.1016/j.chom.2016.05.003.

57. Krishnaveni R, Rathod V, Thakur MS, Neelgund YF. Transformation of L-tyrosine to L-DOPA by a novel fungus, Acremonium rutilum, under submerged fermentation. Curr Microbiol. 2009;58(2):122-8. https://doi. org/10.1007/s00284-008-9287-5.

58. Fraga-Silva TFDC, Mimura LAN, Leite LDCT, Borim PA, Ishikawa LLW, Venturini J, Arruda MSPD, Sartori A. Gliotoxin aggravates experimental autoimmune encephalomyelitis by triggering neuroinflammation. Toxins. 2019;11(8):443. https://doi.org/10.3390/toxins11080443.

59. Gonzalez-Menendez V, Martin J, Siles JA, Gonzalo-Tejero MR, Reyes F, Platas G, Tormo JR, Genilloud O. Biodiversity and chemotaxonomy of Preussia isolates from the Iberian Peninsula. Mycol Progress. 2017;16:713-28. https://doi.org/10.1007/s11557-017-1305-1.

60. Liew WP, Mohd-Redzwan S. Mycotoxin: Its impact on gut health and microbiota. Front Cell Infect Microbiol. 2018;8:60. https://doi.org/10.3389/ fcimb.2018.00060.

61. Leng J, Proudman C, Darby A, Blow F, Townsend N, Miller A, Swann J. Exploration of the fecal microbiota and biomarker discovery in Equine Grass Sickness. J Proteome Res. 2018;17(3):1120-8. https://doi.org/10. 1021/acs.jproteome.7b00784.

62. Sánchez Márquez S, Bills GF, Herrero N, Zabalgogeazcoa I. Non-systemic fungal endophytes of grasses. Fungal Ecol. 2012;5(3):289-97. https://doi. org/10.1016/j.funeco.2010.12.001.

63. Bakker MG. A fungal mock community control for amplicon sequencing experiments. Mol Ecol Resour. 2018;18(3):541-56. https://doi.org/10. 1111/1755-0998.12760.

64. O'Donnell K, Cigelnik E. Two divergent intragenomic rDNA ITS2 types within a monophyletic lineage of the fungus Fusarium are nonorthologous. Mol Phylogenet Evol. 1997;7:103-16. https://doi.org/10.1006/mpev. 1996.0376.

65. Strati F, Di Paola M, Stefanini I, Albanese D, Rizzetto L, Lionetti P, Calabrò A, Jousson O, Donati C, Cavalieri D, De Filippo C. Age and gender affect the composition of fungal population of the human gastrointestinal tract. Front Microbiol. 2016;7:1227. https://doi.org/10.3389/fmicb.2016.01227.

66. Martin R, Dombrowski J. Isolation and identification of fungal endophytes from grasses along the Oregon coast. Am J Plant Sci. 2015;6:3216-30. https://doi.org/10.4236/ajps.2015.619313.

67. Edinburgh Genomics. https://genomics.ed.ac.uk/ Accessed 1 June 2021.

68. Op De Beeck M, Lievens B, Busschaert P, Declerck S, Vangronsveld J, Colpaert JV. Comparison and validation of some ITS primer pairs useful for fungal metabarcoding studies. PLoS One. 2014;9(6):e97629. https:// doi.org/10.1371/journal.pone.0097629.

69. R Core Team (2019). R: A language and environment for statistical computing. R Foundation for Statistical Computing, Vienna, Austria. URL https://www.R-project.org/.

70. McMurdie PJ, Holmes S. Phyloseq: an R package for reproducible interactive analysis and graphics of microbiome census data. PLOS ONE. 2013;8(4): e61217. https://doi.org/10.1371/journal.pone.0061217.

71. Paulson JN, Stine OC, Bravo HC, Pop M. Differential abundance analysis for microbial marker-gene surveys. Nat Methods. 2013;10(12):1200-2. https://doi.org/10.1038/nmeth.2658.

72. Oksanen J, Blanchet FG, Friendly M, Kindt R, Legendre P, McGlinn D, Minchin PR, O'Hara RB, Simpson GL, Solymos P, Henry M, Stevens H, Szoecs E, Wagner H. Vegan: community ecology package. R package version 2.5-6 2019. https://CRAN.R-project.org/package=vegan.

73. Paradis E, Schliep K. ape 5.0: an environment for modern phylogenetics and evolutionary analyses in R. Bioinformatics. 2019;35(3):526-8. https:// doi.org/10.1093/bioinformatics/bty633. 
74. Wickham H. ggplot2: Elegant graphics for data analysis. New York: Springer-Verlag; 2016.

75. Rohart F, Gautier B, Singh A, Lê Cao KA. mixOmics: an R package for 'omics feature selection and multiple data integration. PLoS Comput Biol. 2017;13(11): e1005752. https://doi.org/10.1371/journal.pcbi.1005752.

76. Love MI, Huber W, Anders S. Moderated estimation of fold change and dispersion for RNA-seq data with DESeq2. Genome Biol. 2014;15(12):550. https://doi.org/10.1186/s13059-014-0550-8.

77. Chen J. GUniFrac: Generalized UniFrac Distances. R package version 1.1 2018. https://CRAN.R-project.org/package=GUniFrac.

78. Wickham H, François R, Henry L, Müller K. dplyr: a grammar of data manipulation. R package version 0.8.5. 2020. https://CRAN.R-project.org/ package $=$ dplyr.

79. Gweon HS, Oliver A, Taylor J, Booth T, Gibbs M, Read DS, Griffiths RI, Schonrogge K. PIPITS: an automated pipeline for analyses of fungal internal transcribed spacer sequences from the Illumina sequencing platform. Methods Ecol Evol. 2015;6(8):973-80. https://doi.org/10.1111/2041-210X. 12399.

80. Abarenkov K, Zirk A, Piirmann T, Pöhönen R, Ivanov F, Nilsson RH, Kõljalg U. UNITE retrained 04.02.2020.tar.gz; 2020: UNITE USEARCH/UTAX release for Fungi. Version 04.02.2020. UNITE Community. https://doi.org/ $10.15156 / \mathrm{BIO} / 786375$
81. Callahan B, Davis N. Introduction to decontam. 2018. https://benjjneb. github.io/decontam/vignettes/decontam_intro.html.

82. Karagiannis-Voules DA, Biedermann P, Ekpo UF, Garba A, Langer E, Mathieu E, Midzi N, Mwinzi P, Polderman AM, Raso G, Sacko M, Talla I, Tchuenté LA, Touré S, Winkler MS, Utzinger J, Vounatsou P. Spatial and temporal distribution of soil-transmitted helminth infection in sub-Saharan Africa: a systematic review and geostatistical meta-analysis. Lancet Infect Dis. 2015;15(1):74-84. https://doi.org/10.1016/S1473-3099(14)71004-7.

83. Chen Y, Yang F, Lu H, Wang B, Chen Y, Lei D, Wang Y, Zhu B, Li L. Characterization of fecal microbial communities in patients with liver cirrhosis. Hepatology. 2011;54:562-72. https://doi.org/10.1002/hep.24423.

84. Xu M, Jiang Z, Huang W, Yin J, Ou S, Jiang Y, Meng L, Cao S, Yu A, Cao J, Shen Y. Altered gut microbiota composition in subjects infected with Clonorchis sinensis. Front Microbiol. 2018;9:2292. https://doi.org/10.3389/ fmicb.2018.02292.

\section{Publisher's Note}

Springer Nature remains neutral with regard to jurisdictional claims in published maps and institutional affiliations.
Ready to submit your research? Choose BMC and benefit from:

- fast, convenient online submission

- thorough peer review by experienced researchers in your field

- rapid publication on acceptance

- support for research data, including large and complex data types

- gold Open Access which fosters wider collaboration and increased citations

- maximum visibility for your research: over $100 \mathrm{M}$ website views per year

At BMC, research is always in progress.

Learn more biomedcentral.com/submissions 